relation zwischen Pyrethroiden im Blut und Beschwerden eher gefunden werden, aber aufgrund des rapiden Metabolismus der Pyrethroide ist ein solches Biomonitoring derzeit nur bei Intoxikationen möglich.

\section{Danksagung:}

Die Autoren möchten sich herzlich beim Deutschen Schädlingsbekampferverband (DSV) und insbesonders bei Herrn Schürmann für die Zusammenarbeit bedanken.

\section{Literatur:}

[1] Aldridge, W. N.: An assessment of the toxicological properties of pyrethroids and their neurotoxicity. Critical Reviews in Toxicology 21 (1990) 89-104.

[2] Appel, K. E., und Gericke, S.: Zur Neurotoxizität und Toxikokinerik von Pyrethroiden. Bundesgesundhbl. 36, 6 (1993) 219-228.
[3] Eadsforth, C. V., and Baldwin, M. K.: Human dose-excretion studies with the pyrethroid insecticide cypermethrin. Xenobiotica 13 (1983) 67-72.

[4] Wollen, B. H., Marsh, J. R., Laird, W. J. D., and Lesser, J. E.: The metabolism of cypermethrin in man: differences in urinary meta bolite profiles following oral and dermal administration. Xenobiotica 22 (1992) 983-991.

[5] Leng, G., Leng, A., Kühn, K.-H., Lewalter, J., and Pauluhn, J.: Human dose-excretion studies with the pyrethroid insecticide cyfluthrin: urinary metabolite profile following inha lation. Xenobiotica 27 (1997) 1272-1283.

[6] Kühn, K.-H., Leng, G., Bucholski, K. A., Dunemann, L., and Idel, H.: Determination of pyrethroid metabolites in human urine by capillary gas chromatography-mass spectrometry. Chromatographia 43 (1996) 285-292

[7] Leng, G., Kühn, K.-H., and Idel, H.: Biological monitoring of pyrethroids in blood and pyrethroid metabolites in urine: applications and limirations. Sci. Total. Environ. 199 (1997) 173-181.
[8] He, F., Sun, J., Han, K., Wu, Y., Yao, P. Wang, S., and Liu, L.: Effects of pyrethroid insecticides on subjects engaged in packaging pyrethroids. Br. J. Ind. Med. 45 (1988) 548-551.

[9] He, F., Wang, S., Liu, L., Chen, S., Zhang, Z. and Sun, J.: Clinical manifestations and diagnosis of acute pyrethroid poisoning. Arch. Toxicol. 63 (1989) 54-58.

[10] Kolmodin-Hedman, B., Akerblom, M., Flato S., and Alex, G.: Symptoms in Forestry Workers Handling Conifer Plants Treated with Permethrin. Bull. Environ. Contam. Toxicol. 55 (1995) 487-493.

[11] Zhang, Z., Sun, J., Chen, S., Wu, Y., and He F.: Levels of exposure and biological monitoring of pyrethroids in sprayman. $\mathrm{Br}$. J. Ind Med. 48 (1991) 82-86.

Prof. Dr. med. G. Leng, B. Wieseler, Dr. rer. nat. K.-H. Kühn, Prof. Dr. med. H. Idel, Institut fü Hygiene der Heinrich-Heine-Universität Düsseldorf, Moorenstraße 5, 40225 Düsseldorf

\title{
Aufdeckung von Ausbrüchen bei Infektionen mit enterohämorrhagischen Escherichia coli (EHEC) O157
}

\section{Bericht über eine Zusammenarbeit von Landesinstituten des Öffentlichen Gesundheitsdienstes mit dem Robert Koch-Institut}

Enterohämorrhagische $E$. coli (EHEC) sind als neuartige Krankheitserreger seit 1982 bekannt. EHEC verursachen beim Menschen wäßrige bis wäßrig-blutige Durchfallerkrankungen. Besonders bei Kindern und bei alten Menschen können in Folge einer EHEC-Infektion Nierenschädigungen in Form eines Hämolytisch Urämischen Syndroms (HUS) auftreten, die lebensbedrohlich verlaufen können [1].

EHEC kommen in Deutschland und in vielen anderen Ländern endemisch vor Das natürliche Reservoir der EHEC sind landwirtschaftliche Nutztiere, vor allem große und kleine Wiederkäuer, die diese Keime mit ihrem Kot ausscheiden können, ohne selbst erkrankt zu sein. Unter den EHEC wurden solche der Typen O157:H7 und O157:H- bei Ausbrüchen weltweit bisher am häufigsten isoliert $[2,3])$. Untersuchungen zeigten, daß EHEC O157 eine hohe Infektiosität für den Menschen besitzen. Diese wird auf die für den Menschen niedrige In- fektionsdosis ( $<100$ lebende Keime) und die Robustheit der Erreger gegenüber physikalisch-chemischen Stressbedingungen zurückgeführt [2].

Obwohl die bisher in Deutschland aufgetretenen Fälle von EHEC-Infektionen überwiegend als sporadische Ereignisse registriert wurden, ist anzuneh men, daß in vielen Fällen kleinere und größere Ausbrüche an Erkrankungen vorgelegen haben [4-6]. Ein Ausbruch besteht nach Definition der WHO bereits dann, wenn mehr als eine Person durch den gleichen Ausbruchsstamm (Klontyp) eines Erregers infiziert wird [2]. Ausbrüche können jedoch nur entdeckt werden, wenn in Fällen von $\mathrm{Er}$ krankungen Umgebungsuntersuchungen durchgeführt werden. Dies ist bei der überwiegenden Zahl der als sporadisch registrierten EHEC-Infektionen noch eher die Ausnahme als die Regel [4].

In dieser Arbeit sollen Beispiele für erfolgreich durchgeführte Umgebungsun- tersuchungen zur Aufklärung von Ausbrüchen und möglichen Infektketten bei EHEC-Infektionen dargestellt werden. Bei diesen Untersuchungen wurden EHEC-O157-Isolate aus einem vermuteten Ausbruchsgeschehen an Hand der von den Erregern gebildeten Vero (Shiga)-Toxine sowie anderer Virulenzfaktoren miteinander verglichen. $\mathrm{Zu}$ sätzlich wurden an den Isolaten vergleichende genetische Untersuchungen mit Hilfe der Pulsfeld-Gelelektrophorese (PFGE) vorgenommen. Die Kombination von klassischer Erregertypisierung und dem »genetischen Fingerabdruck * ist gut geeignet, um die klonale Identität von EHEC-Isolaten bei Ausbrüchen und Umgebungsuntersuchungen $\mathrm{zu}$ überprüfen [7].

Bei der genetischen Untersuchung mit Hilfe der PFGE wird die Gesamt-DNA der Bakterien mit selten schneidenden Restriktionsenzymen ( $z$. B. XbaI) enzymatisch verdaut, und die dadurch entstehenden DNA-Fragmente werden 
gel-elektrophoretisch aufgetrennt. Aus der Spaltung von $E$. coli-DNA mit dem Restriktionsenzym $X b a \mathrm{I}$ entstehen $c a$. 20 Banden von 10 bis $700 \mathrm{~kb}$ Größe. Je nach Verwandtschaft der E. coli-Isolate ergeben sich hierbei sehr unterschiedliche bis völlig homogene Bandenmuster [7]. Nach Durchführung der Gelelektrophorese lassen sich die nach Fragmentgröße aufgetrennten Bandenmuster durch Anfärbung des Gels mit Ethidiumbromid fotografisch dokumentieren und vergleichen. Von Tenover et al. (7) wurden Kriterien zur Interpretation von PFGE-Mustern bei der Untersuchung von Ausbrüchen aufgestellt. Hiernach liegt bei einer völligen Übereinstimmung der Bandenmuster eine gesicherte Verwandtschaft zwischen den Isolaten (gleicher Klontyp) vor, das heißt die Isolate stammen mit hoher Wahrscheinlichkeit aus einem Ausbruchsgeschehen. Bei nur geringen Abweichungen (2-3 Banden Unterschiede) ist die Verwandtschaft der Isolate wahrscheinlich. Abweichungen von sieben und mehr Banden lassen auf Erreger schließen, die mit hoher Sicherheit nicht aus einem gemeinsamen Ausbruchsgeschehen stammen.

\section{Fünf dokumentierte Aus- brüche durch EHEC O157}

Es werden fünf Fälle von EHEC-O157Infektionen beschrieben, bei denen durch Umgebungsuntersuchungen ein Ausbruchsgeschehen aufgedeckt wurde. In allen Fällen lagen Infektionshäufungen mit dem gleichen Klontyp vor. Zwischen den fünf untersuchten Ausbrüchen bestand jedoch keine ersichtliche epidemiologische Beziehung. Die Untersuchungen zu den Fällen 1 und 2 wurden am Landeshygieneinstitut Mecklenburg-Vorpommern, zu den Fällen 3 und 4 an zwei Berliner Krankenhäusern und zum Fall 5 am Landesinstitut für den öffentlichen $\mathrm{Ge}$ sundheitsdienst Nordrhein-Westfalen durchgeführt. Die genetische Typisierung der EHEC-O157-Isolate erfolgte am Robert Koch-Institut (Berlin). Die Ergebnisse zu diesen Fällen sind in $\mathrm{Ta}$ belle 1 und in der Abbildung 1 dokumentiert.

Fall 1 betrifft ein einjähriges Mädchen, daßs wegen schweren Durchfalls hospitalisiert wurde. Aus der Stuhlprobe des Kindes konnten EHEC O157:H7 isoliert werden. Bei der Umgebungsuntersuchung in der Familie des Kindes konnten der Vater und der Bruder des Mädchens als asymptomatische Aus- scheider des gleichen Epidemieklons identifiziert werden.

Fall 2 betrifft einen zweijährigen Jungen mit unkomplizierter, nicht hämorrhagischer Enteritis, bei dem aus dem Stuhl EHEC O157:H7 angezüchtet werden konnten. In der Familie (sechs Stuhlproben) und im Kindergarten (16 Stuhlproben) wurden Umgebungsuntersuchungen vorgenommen. EHEC O157:H7 konnten nur in der Stuhlprobe des gesunden Großvaters des Kindes, der auf einem Bauernhof lebt, nachgewiesen werden. Beide EHEC-O157:H7-Isolate ließen sich dem gleichen Klontyp zuordnen. Die Untersuchung von Kotproben der auf dem Hof gehaltenen Rinder auf EHEC O157 verlief negativ.

Fall 3 betrifft zwei Kleinkinder, die zur gleichen Zeit in einem Berliner Krankenhaus stationär wegen einer Lymphadenitis bzw. einer Meningokokkensepsis behandelt wurden. Zwischen den Kindern bzw. ihren Familien bestanden keine Kontakte. Beide Kinder waren in getrennten Zimmern des Krankenhauses untergebracht, wurden jedoch vom selben Pflegepersonal betreut. Im Rahmen von Kontrolluntersuchungen wurden aus den Stühlen beider Kinder EHEC O157:H- eines Klontyps isoliert. Bei keinem der Kinder kam es zur Entwicklung einer Durchfallsymptomatik bzw. eines HUS.

Fall 4 betrifft einen vier Jahre alten Jungen, der mit einem enteropathischen HUS in der Kindernephrologie eines Berliner Krankenhauses stationär behandelt wurde. Aus dem Stuhl des Kindes konnten EHEC O157:H7 isoliert werden. Die Untersuchung der Familie des Patienten führte zur Isolierung des gleichen Klontyps von O157:H7 aus dem Stuhl seines achtjährigen beschwerdefreien Bruders.

Fall 5 betrifft einen ca. einjährigen Jungen, bei dem ein Durchfallgeschehen begleitet von hohem Fieber vorlag. Bei der mikrobiologischen Untersuchung konnten EHEC O157:H- aus dem Stuhl angezüchtet werden. Vom zuständigen Medizinalaufsichtsamt wurden Kontrolluntersuchungen in der Familie veranlaßt. Hierbei stellte sich heraus, daß

Tabelle 1: Falldaten zu fünf dokumentierten Ausbrüchen mit EHEC 0157

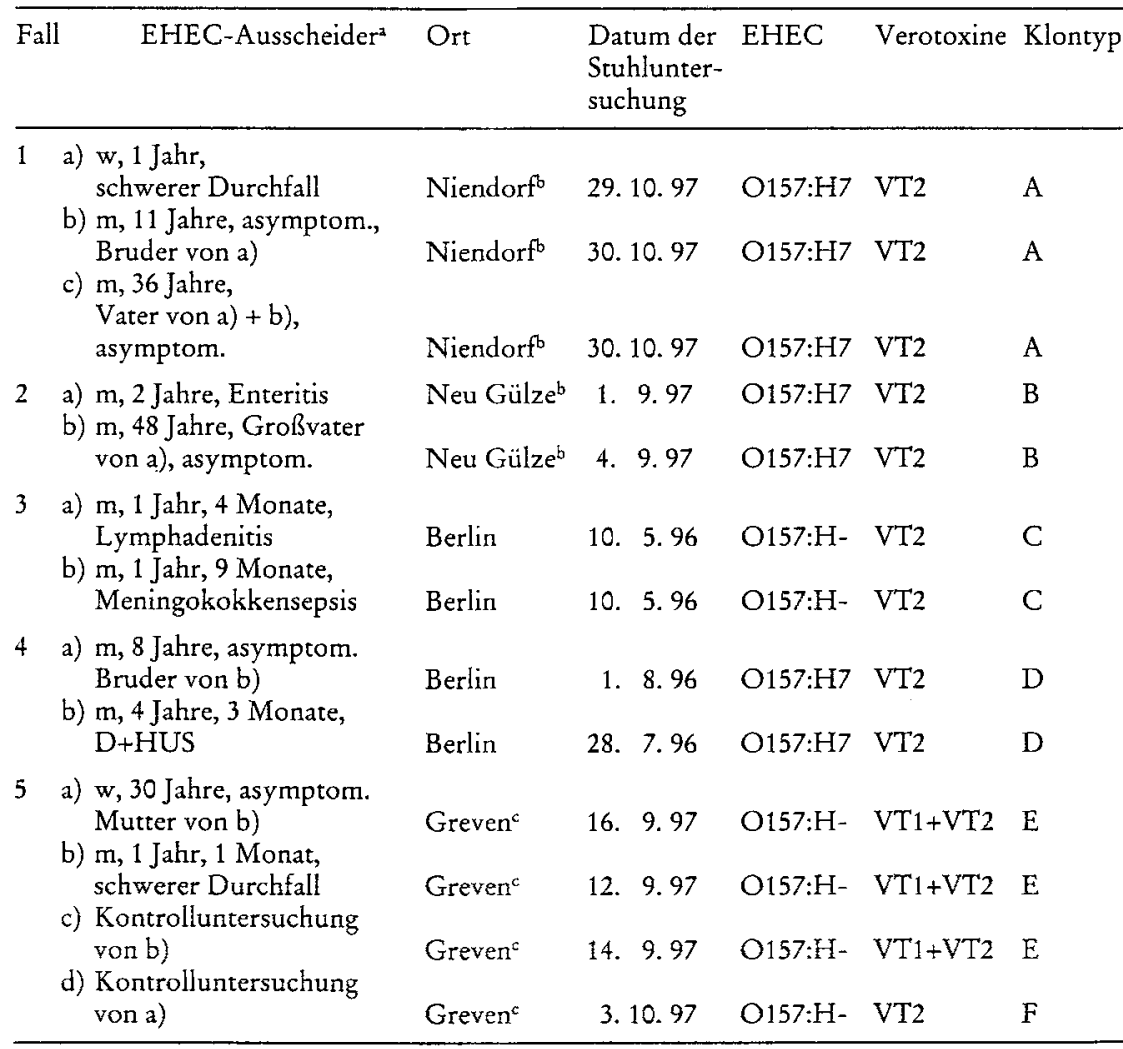

a) $w=$ weiblich, $m=$ männlich, asymptom. = asymptomatischer Ausscheider von EHEC O157, D+HUS = enteropathisches Hämolytisch-Urämisches Syndrom;

b) Gesundheitsamt Ludwigslust;

c) Gesundheitsamt Ibbenbühren 


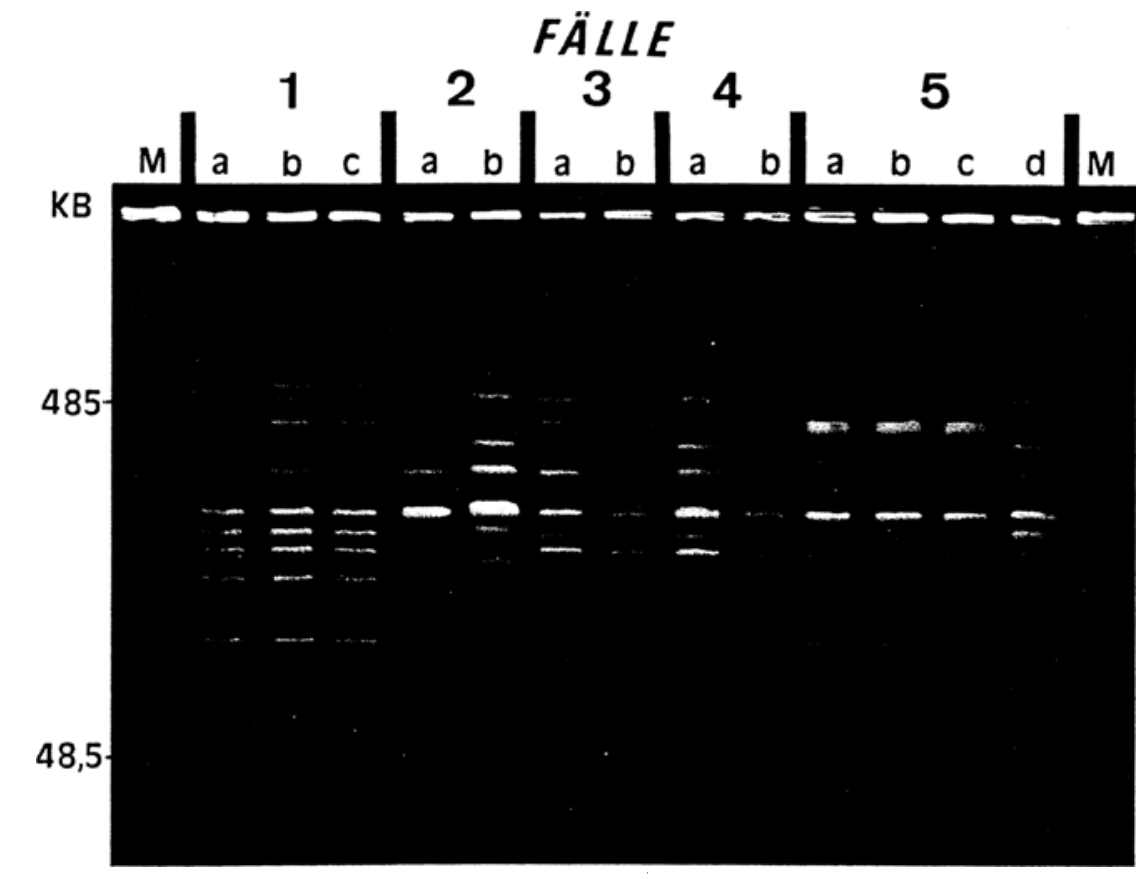

Abbildung 1: Fotografische Darstellung der durch PFGE aufgetrennten XbaI-Bandesmuster von 13 EHEC-O157-Isolaten aus insgesamt fünf Ausbrüchen.

Spuren $\mathrm{M}=$ Molekulargewichtsstandard, die Größe der aufgetrennten XbaI Fragmente ist in Kilobasen (KB) angegeben. Die einzelnen Angaben zu den EHEC-Isolaten (Spuren 1 a bis $5 \mathrm{~d}$ ) sind aus Tabelle $1 \mathrm{zu}$ entnehmen.

die 30jährige Mutter des Kindes symptomlose Ausscheiderin von EHEC O157:H- war. Die EHEC-Isolate der Mutter und des Kindes gehörten zum gleichen Klontyp. Bei einer erneuten Kontrolluntersuchung konnte 14 Tage später bei der beschwerdefreien Mutter des Kindes erneut O157: $\mathrm{H}$ - isoliert werden. Jedoch gehörte dieses Isolat zu einem anderen Klontyp, als die ursprünglich beim Kind und seiner Mutter isolierten EHEC. Es ist daher anzunehmen, daß es sich um eine erneute Infektion der Mutter mit einem anderen O157 Klon handelte, bzw. daß beide O157 Klone von der Mutter ausgeschieden wurden, aber nicht in allen Untersuchungen nachgewiesen wurden.

\section{Schlußfolgerungen}

In Zusammenarbeit zwischen Landesinstituten des Öffentlichen Gesundheitsdienstes, Berliner Krankenhäusern und dem Robert Koch-Institut konnten fünf voneinander unabhängige Ausbrüche durch EHEC O157 aufgedeckt werden. In allen fünf Fällen erwies sich die Untersuchung der engen Kontaktpersonen der Patienten als ein geeignetes Mitte zur Aufdeckung von klinisch unauffälligen Ausbrüchen mit symptomlosen Ausscheidern von EHEC O157.
Nach Angaben der WHO sind folgende Hauptübertragungswege der EHEC bekannt: 1. Verzehr von EHEC-kontaminierten Nahrungsmitteln, 2. direkte Übertragung von Mensch zu Mensch (fäkal-oral), 3. Übertragung durch Kontakte mit Ausscheidertieren (fäkal-oral) und 4. Übertragung durch kontaminiertes Trink- und Badewasser [2, 3]. In keinem der hier beschriebenen Fälle konnte ein EHEC-kontaminiertes Lebensmittel als ursprüngliche Infektionsquelle identifiziert werden. Allerdings sind EHEC-kontaminierte Lebensmittel als mögliche Infektionsursache nicht völlig auszuschließen, da zwischen Verzehr, dem Auftreten einer Durchfallerkrankung und einer Stuhluntersuchung in der Regel mehrere Tage vergehen, so daß verdächtige Lebensmittelproben für eine Untersuchung oft nicht mehr zur Verfügung stehen bzw. nicht mehr erinnerlich sind.

Neben der Übertragung durch Lebenswerden, daß der Übertragung von EHEC von Mensch zu Mensch eine wichtige Bedeutung zukommt $[5,6,8]$. Hierbei spielt die niedrige minimale Infektionsdosis und die Säureresistenz dieser Krankheitserreger eine erhebliche Rolle [2]. mittel konnte in vielen Fällen gezeigt
Bei den hier dokumentierten Ausbrüchen fällt auf, daß ältere Kontaktpersonen in der Umgebung der erkrankten Kleinkinder oft als symptomlose Ausscheider des gleichen Krankheitskeimes auftraten. Das Auftreten von symptomlosen EHEC-Ausscheidern deutet auf eine mögliche bestehende individuelle Immunität gegenüber EHEC als Krankheitserreger hin. Untersuchungen aus Kanada konnten zeigen, daß bei potentiell EHEC-exponierten Personen der Landbevölkerung (regelmäßiger Verzehr von Rohmilch, Kontakte mit EHEC-Ausscheidertieren) eine hohe Zahl an asymptomatischen EHECAusscheidern anzutreffen ist [9]. $\mathrm{Zu}$ sammengefaßt zeigen diese Befunde auch, daß der Weiterverbreitung von EHEC-Infektionen durch asymptomatische Ausscheider eine größere Bedeutung zukommt, als es bisher angenommen wurde.

Die hier stichprobenartig aufgedeckten Ausbrüche an EHEC O157-Infektionen, die nur in Einzelfällen mit einer Erkrankung einhergingen, deuten darauf hin, daß möglicherweise bei einer größeren Zahl von Einzelfällen von Erkrankungen ein unerkanntes Ausbruchsgeschehen vorliegt. Interessanterweise waren die hier beschriebenen Ausbrüche nicht durch eine Häufung von Erkrankungen gekennzeichnet, sondern durch das Auftreten von asymptomatischen Ausscheidern in der engen Umgebung der Patienten. Vergleichbare Befunde ergaben sich auch bei Umgebungsuntersuchungen, die in Bayern mit Einführung der Meldepflicht für EHECAusscheider durchgeführt wurden [10] Daher sollte auch bei Fällen von EHECInfektionen ohne eine beobachtete Häufung von Erkrankungen durch Umgebungsuntersuchungen gegebenenfalls nach einem Ausbruchsgeschehen ermittelt werden.

\section{Antiepidemische Maßnahmen bei EHEC-Infektionen in Mecklenburg-Vorpommern}

Bericht aus dem Landeshygieneinstitut (Rostock)

Neben anderen humanpathogenetisch bedeutsamen $E$. coli-Typen werden in Mecklenburg-Vorpommern Fälle von EHEC-Infektionen wie üblich in der Form gemeldet, daß der Nachweis des entsprechenden $E$. coli-Serovars vom behandelnden Arzt und vom Labordiagnostiker dem zuständigen Gesundheitsamt mitgeteilt wird, das dann wie- 
derum das Landeshygieneinstitut informiert. Unklare Befunde, wie z. B. Verotoxinnachweis in der Stuhlprobe ohne Erregeridentifizierung, sind nach Möglichkeit vom Landeshygieneinstitut nachzukontrollieren und abzuklären. Wie bei jeder anderen Form der infektiösen Enteritis werden nach Meldung eines EHEC-Erregers vom Gesundheitsamt die notwendigen antiepidemischen Maßnahmen eingeleitet und koordiniert, das betrifft vor allem

\section{1. das sofortige Aufsuchen des betroffe-} nen Patienten und seiner Familie sowie seines Umfeldes (Kindereinrichtung, Seniorenheim);

2. die umgehende Einleitung von bakteriologischen Stuhluntersuchungen in den betroffenen Familien bzw. Gemeinschaften zum Erregernachweis und zum auffinden symptomloser Keimträger;

3. die Übermittlung der EHEC-Befunde an das zuständige Veterinärund Lebensmittelüberwachungsamt zur Einleitung bakteriologischer Kotuntersuchungen in verdächtigen Tierbeständen, sofern sich ein Hinweis auf epidemiologische Zusammenhänge ergeben hat.

Eine wesentliche Voraussetzung für eine hohe Aufklärungsrate bei EHECErkrankungen und anderen Infektionen ist nach unserer Erfahrung die kontinuierliche, intensive Zusammenarbeit und der Informationsaustausch zwischen Landeshygieneinstitut, Gesundheitsämtern und Veterinär- und Lebensmittelüberwachungsämtern. Der Informationsaustausch erfolgt in Form von Fachgesprächen zwischen kompetenten Mitarbeitern der genannten Institutionen und in Form einer jährlich stattfindenden Tagung, zu der alle Amtsärzte und Amtstierärzte bzw. deren Mitarbeiter eingeladen werden und bei der über aktuelle human- und veterinärmedizinisch interessierende Themen referiert wird. Hierbei waren EHEC-Infektionen in den letzten Jahren immer wieder ein $\mathrm{Ta}$ gesordnungspunkt.

Weiterhin sind von der Arbeitsgruppe Epidemiologie des Landeshygieneinstitutes "Empfehlungen für Schutzmaßnahmen beim Auftreten übertragbarer
Krankheiten « zusammengestellt worden [11]. Die Publikation enthält neben antiepidemischen Maßnahmen auch Angaben zum Erreger, zur Klinik, Prophylaxe und zur Therapie und Labordiagnostik bei Infektionen. Die Empfehlungen werden laufend aktualisiert, den Amtsärzten des Landes zur Verfügung gestellt und können von jedem Interessierten erworben werden. Durch die 1995 erschiene Publikation "Enterohämorrhagische $E$. coli (EHEC) - ein neuer Erreger « im Ärzteblatt Mecklenburg-Vorpommern konnten viele Ärzte auf diese Problematik aufmerksam gemacht werden [12].

Im Jahr 1997 wurden in MecklenburgVorpommern 22 EHEC-Infektionen gemeldet. Gegenüber 1996 (elf Fälle) ist ein Anstieg auf das Doppelte zu verzeichnen. Die 1997 angezeigren EHECInfektionen betrafen $17 \mathrm{E}$. coli-O157Infektionen und einen Nachweis von EHEC O26. Bei vier Personen wurde ein Verotoxinnachweis im Stuhl gemeldet, ohne daß ein EHEC angezüchtet werden konnte. Die beobachtete $\mathrm{Zu}$ nahme ist unseres Erachtens vor allem auf die verbesserte Labordiagnostik dieser Infektionen zurückzuführen, zumal im Jahr 1997 keine der gefürchteten klinischen Manifestationen wie HUS oder TTP (Thrombotisch-thromozytopenische Purpura) im Land beobachtet wurden.

Trotz der beschriebenen Bemühungen aller Beteiligten um eine hohe Aufklärungsrate bei EHEC-Infektionen konnte erst im IV. Quartal 1997 durchgesetzt werden, daß zu jedem gemeldeten EHEC-Ausscheider in Mecklenburg-Vorpommern auch eindeutige bakteriologische Befunde ( $E$. coli-Serovar, Verotoxinnachweis im Stuhl und beim Erreger) sowie exakte klinische Angaben mitgeteilt werden.

\section{Literatur:}

[1] Beutin, L.: Infektionen mit enterohämorrhagischen Escherichia coli (EHEC). Bundesgesundhbl. 39, 11 (1996) 426-429.

[2] World Health Organization: Prevention and control of enterohaemorrhagic Escherichia coli (EHEC) infections. Report of a WHO consultation. Geneva, Switzerland, 28 April1 May, 1997. WHO/FSF/FOS/97.6 (1997).

[3] World Health Organization: WHO consultation on selected emerging foodborne diseases.
Berlin, Germany, 20-24 March, 1995. WHO/CDS/VPH/95.142 (1995).

[4] Robert Koch-Institut (Hrsg.): Zum Aufbau einer Surveillance für enterohämorrhagische E. coli (EHEC) in Deutschland. Epidemiologisches Bullerin 39/97, 26. 9. 1997.

[5] Bockemühl, J., Karch, H., und Tschäpe, H.: Infektionen des Menschen durch enterohämorrhagische Escherichia coli (EHEC) in Deutschland, 1996. Bundesgesundhbl. 40, 6 (1997) 194-197.

[6] Beutin, L., Zimmermann, S., und Gleier, K.: Zur Epidemiologie und Diagnostik von Infektionen durch enterohämorrhagische $E$. coli (EHEC) in der Bundesrepublik Deutschland. Mikrobiologisch nachgewiesene Fälle von Infektionen durch EHEC bei Menschen in den Jahren 1993 bis 1995. Bundesgesundhbl. 39,9 (1996) 326-331.

[7] Tenover, F. C., Arbeit, R. D., Goering, R. V., Mickelsen, P. A., Murray, B. E., Persing, D. H., and Swaminathan, B.: Interpreting chromosmal DNA restriction patterns produced by pulsed-field gel electrophoresis: Criteria for strain typing. J. Clin. Microbiol. 33 (1995) 2233-2239.

[8] Reida, P., Wolff, M., Pöhls, H.-W., Kuhlmann, W., Lehmacher, A., Aleksic, S., Karch, H., and Bockemühl, J.: An outbreak due to enterohemorrhagic Escherichia coli in a children day care center characterized by person to person transmission and environmental contamination. Zbl. Bakt. 281 (1994) 534-543.

[9] Wilson, J. B., Clarke, R. C., Renwick, S. A. Rahn, K., Johnson, R. P., Karmali, M. A., Lior, H., Alves, D., Gyles, C. L., Sandhu, K. S., McEwen, S. A., and Spika, J. S.: Vero cytotoxigenic Escherichia coli infection in dairy farm families. J. Infect. Dis. 174 (1996) 1021-1027.

[10] Pulz, M.: Zur Epidemiologie und aktuellen Bedeutung enterohämorrhagischer Escherichia coli (EHEC) in Nordbayern (1996). Gesundheitswesen 59 (1997) 656-660.

[11] Hülße, C., Kober, P., Littmann, M., und Pohl, U.: Empfehlungen für Schutzmaßnahmen bei Auftreten übertragbarer Krankheiten. Informationen zur Epidemiologie, Klinik, Prophylaxe und Therapie ausgewählter Infektionskrankheiten. AG »Infektionsepidemiologie des Landeshygieneinstitutes MecklenburgVorpommern (1997).

[12] Kontny, I., und Hülße, C.: Enterohämorrhagische E. coli (EHEC) - ein neuer Erreger? Arzteblatt Mecklenburg-Vorpommern 9 (1995) 422

Dr. Lothar Beutin, Fachbereich Bakteriologie, Fachgebiet für Enterobacteriaceac und pathogene Escherichia coli, Robert Koch-Instirut; Dr. I. Kontny, Abteilung Mikrobiologie, Landeshygieneinstitut Mecklenburg-Vorpommern, Rostock; Dr. S. Kuttner-May, Landesinstitut für den Öffentlichen Gesundheitsdienst NRW, Münster

\section{Korrespondenzanschrift:}

Dr. Lothar Beutin, Robert Koch-Institut, Nordufer 20, D-13353 Berlin 